\title{
Identification of calcium-modulating cyclophilin ligand as a human host restriction to HIV-1 release overcome by Vpu
}

\author{
Vasundhara Varthakavi ${ }^{1}$, Ellen Heimann-Nichols ${ }^{1}$, Rita M Smith ${ }^{1}$, Yuehui Sun ${ }^{1}$, Richard J \\ Bram $^{2}$, Showkat Ali ${ }^{3}$, Jeremy Rose ${ }^{3}$, Lingmei Ding ${ }^{3}$, and Paul Spearman ${ }^{3}$ \\ 1 Department of Pediatrics, Division of Infectious Diseases, Vanderbilt University School of Medicine, 1161 \\ 21st Avenue South, D-7235 MCN, Nashville, Tennessee 37232-2581, USA \\ 2 Department of Pediatric and Adolescent Medicine, Division of Pediatric Research Laboratories, Mayo \\ Clinic, Mayo Medical School, 200 First Street Southwest, Rochester, Minnesota 55905, USA \\ 3 Department of Pediatrics, Division of Infectious Diseases, Emory University School of Medicine, 2015 \\ Upper Gate Drive, Atlanta, Georgia 30322, USA
}

\begin{abstract}
The HIV-1 Vpu protein is required for efficient viral release from human cells. For HIV-2, the envelope (Env) protein replaces the role of Vpu. Both Vpu and HIV-2 Env enhance virus release by counteracting an innate host-cell block within human cells that is absent in African green monkey (AGM) cells. Here we identify calcium-modulating cyclophilin ligand (CAML) as a Vpu-interacting host factor that restricts HIV-1 release. Expression of human CAML (encoded by CAMLG) in AGM cells conferred a strong restriction of virus release that was reversed by Vpu and HIV-2 Env, suggesting that CAML is the mechanistic link between these two viral regulators. Depletion of CAML in human cells eliminated the need for Vpu in enhancing HIV-1 and murine leukemia virus release. These results point to CAML as a Vpu-sensitive host restriction factor that inhibits HIV release from human cells. The ability of CAML to inhibit virus release should illuminate new therapeutic strategies against HIV.
\end{abstract}

$\mathrm{Vpu}$ is an oligomeric type 1 integral membrane protein that has two distinct and separable functions in HIV replication ${ }^{1,2}$. Vpu interacts with CD4 and the host factor human $\beta$-transducin repeat-containing protein ( $\beta$ - TrCP), resulting in degradation of $\mathrm{CD} 4$ via the ubiquitindependent proteolytic apparatus ${ }^{3-6}$. A second function of $\mathrm{Vpu}$ is to enhance viral release from infected human cells, including primary macrophages and lymphocytes 1,7 . In the absence of Vpu, HIV particles fail to detach from the plasma membrane and accumulate in large numbers at the cell surface ${ }^{8}$. In contrast to its role in human cells, Vpu is dispensable for efficient HIV release in simian cell types such as CV-1 and its derivative cell line, Cos-79,10. The effect of $\mathrm{Vpu}$ on virus release is not limited to HIV-1 and is also seen with equine infectious anemia virus (EIAV), Visna virus, HIV-2 and murine leukemia virus (MLV) ${ }^{11,12}$.

\footnotetext{
Correspondence should be addressed to V.V. (vasundhara.varthakavi@vanderbilt.edu) or P.S. (paul.spearman@emory.edu). AUTHOR CONTRIBUTIONS

V.V. designed and performed most aspects of this study. E.H.-N. performed biochemical assays and Y.S. generated the CAML mapping constructs with the support of V.V. R.M.S. and V.V. performed yeast two-hybrid analysis. R.J.B. generated the CAML knockdown plasmids. S.A., J.R. and L.D. conducted the microscopy and EM experiments with the support of P.S. V.V. wrote this manuscript. All authors discussed the results and commented on the manuscript.

Published online at http://www.nature.com/naturemedicine/

Reprints and permissions information is available online at http://npg.nature.com/reprintsandpermissions/
} 
HIV-2 lacks a $v p u$ gene but has evolved a distinct means of promoting particle release that is mediated by its Env protein. Like Vpu, HIV-2ROD10 Env enhances release of several retroviruses including HIV-1, HIV-2 and simian immunodeficiency virus ${ }^{13}$. Both Vpu and HIV-2 Env require a functional recycling endosome to exert their viral release function ${ }^{14}$. These two proteins seem to act on a common pathway, overcoming a restriction to particle release that is present in most human cells but lacking in AGM cells $10,11,14$. The molecular nature of the restriction factor targeted by Vpu and HIV-2 Env in human cells has been uncertain.

Here we identify CAML as a Vpu-sensitive human host restriction to HIV-1 release. CAML is a cyclophilin B-binding integral membrane protein that is required for survival of developing $\mathrm{T}$ cells and for regulation of the $\mathrm{T}$ cell receptor-activated tyrosine kinase $\mathrm{p} 56^{\mathrm{Lck}}$ (refs. 15, 16). CAML is essential for embryonic development in mice ${ }^{16}$ and is required for efficient recycling of epidermal growth factor receptor to the plasma membrane ${ }^{17}$. We found that expression of human CAML rendered simian cells restrictive for viral release in the absence of $\mathrm{Vpu}$. Both Vpu and HIV-2 Env rescued $v p u^{-}$virus release in AGM cells expressing human CAML. Depletion of endogenous CAML in HeLa human cervical cancer cells and human $\mathrm{T}$ lymphocytes by RNA interference (RNAi) eliminated the need for Vpu to elicit efficient HIV and MLV release. These data show that CAML is a human host restriction factor that acts at a late budding step in retroviral replication and is counteracted by the viral Vpu protein.

\section{RESULTS}

\section{Vpu interacts with CAML}

To identify the cellular factor targeted by Vpu in human cells, we screened a restrictive human HeLa complementary DNA library in yeast, using full-length Vpu as bait. Twenty-three percent of the positive clones contained sequences corresponding to a human gene known as CAMLG (Fig. 1a and Supplementary Fig. 1 online). We initially confirmed the Vpu-CAML interaction in a sensitive colorimetric $\beta$-galactosidase ( $\beta$-gal) assay by measuring $\beta$-gal activity in yeast transformed with both full-length Vpu-Gal4 binding domain and either full-length recombinant CAML (amino acids 1-296) fused to the transactivation domain or various truncated recombinant CAMLs fused to the activation domain (Fig. 1a). We noticed strong interactions of $\mathrm{Vpu}$ with $\mathrm{CAML}_{1-296}$, as well with as truncation proteins $\mathrm{CAML}_{1-152}$ and $\mathrm{CAML}_{1-189}$. However, the C-terminal CAML $190-296$ did not interact with Vpu. These data suggest that the hydrophilic N-terminus of human CAML (amino acids 1-152) is sufficient for interaction with $\mathrm{Vpu}$.

The interaction of CAML with Vpu was confirmed by co-immunoprecipitation (co-IP) experiments with antibodies to endogenous CAML in human cells (Fig. 1b-d). Precleared cell lysates from HeLa cells expressing hemagglutinin (HA)-tagged Vpu (VpuHA) and Jurkat E6-1 lymphocytes infected with HIV NL4-3 were incubated with CAML-specific polyclonal or monoclonal antibodies. The resulting complexes were analyzed by immunoblotting with antibodies to HA (Fig. 1b) or Vpu (Fig. 1d). Antibodies to CAML were able to specifically immunoprecipitate a complex of CAML and VpuHA from HeLa cells (Fig. 1b). Similarly, coIP performed in HIV-infected E6-1 cell lysates revealed specific interaction of Vpu with endogenous CAML only when antibodies to CAML were used (Fig. 1d). For normalization purposes, we tested aliquots of representative cell lysates from the co-IP reactions in parallel for the expression of VpuHA (Fig. 1c) or Vpu (Fig. 1e) and actin (Fig. 1c, e). Next, to determine whether Vpu and CAML colocalize in restrictive cells, we performed immunofluorescence microscopy of HeLa cells expressing a biologically active $\mathrm{Vpu}$-fluorescent protein fusion, Vpu-cherry (Supplementary Fig. 1 online). CAML and Vpu-cherry showed substantial colocalization especially in the perinuclear region, with less colocalization on peripheral vesicular structures (Supplementary Fig. 1). 


\section{Human CAML restricts HIV release in simian cells}

We next tested the role of CAML as a restrictive factor in HIV-1 release by expressing human CAML (hCAML) in simian Cos-7 cells (Fig. 2a,b). Permissive Cos-7 cells expressing increasing amounts of HA-tagged hCAML (HA-hCAML) or HA alone were infected with a Vpu deletion mutant pseudotyped with vesicular stomatitis virus G protein (VSV-G) (NLUdel; Fig. 2a). After $2 \mathrm{~d}$, supernatants and cells from three independent experiments were assayed for HIV-1 by p24 ELISA. As expected, Cos-7 cells were efficient in supporting release of NLUdel (Fig. 2a). Vpu did not promote viral release from NLUdel-infected Cos-7 cells (Fig. 2a). However, transfection of Cos-7 cells with increasing amounts of a plasmid expressing HA-hCAML resulted in dose-dependent inhibition of NLUdel release (Fig. 2a). The corresponding increase in HA-hCAML expression in cells and increase in cell-associated Gag was assayed by immunoblotting of cell lysates with antibodies specific for HA or CA (viral capsid) (Fig. 2b). A similar experiment performed with wild-type NL4-3 virus with intact Vpu revealed no effect of hCAML on viral release in Cos-7 cells (Supplementary Fig. 2a,b online).

We assessed the relevance of hCAML to replication of HIV in a multiple-round assay by infecting Cos-7 cells expressing CD4 with NL4-3 or NLUdel (Fig. 2c, d). In this experiment, Cos-7 cells expressing CD4 were transfected with an empty vector or with plasmid expressing hCAML tagged with myc. Both mutant and wild-type viruses replicated efficiently in Cos-7 cells (Fig. 2c). However, in Cos-7 cells expressing hCAML, we noticed significant differences in the production of Vpu deletion mutant virus and NL4-3 virus (Fig. 2c). In these cells, NL4-3 virus production was unaffected, with viral p 24 antigen release steadily increasing over time (Fig. 2c). In contrast, NLUdel virus production was severely impaired at early time points, and diminished amounts of virus remained apparent even at later time points (Fig. 2d). These results suggest that human, but not simian, CAML mediates restriction of $v p u^{-}$HIV-1 release. We extended our analysis to a human osteosarcoma-derived cell line, HOS, which allows for Vpuindependent HIV release ${ }^{18}$. Similar to Cos-7 cells, HOS cell HA-hCAML expression also resulted in strong inhibition of NLUdel release (Supplementary Fig. 3a online). Western blot analysis of CAML expression revealed that low-passage HOS cells from the American Type Culture Collection expressed a reduced amount of CAML compared to the restrictive human HeLa and E6-1 cells (Supplementary Fig. 3b). These results indicate a correlation between CAML expression and the restrictive phenotype in the human cells. We note, however, that CAML expression varied when different sources of HOS cells of less certain passaging history were used (data not shown).

We compared the restrictive effect of hCAML with that of the Vpu-binding protein Ubp, which has previously been shown to inhibit release of HIV-1 in a Vpu-dependent manner ${ }^{19}$. Although hCAML inhibited NLUdel release at each time point tested, inhibition by Ubp was noticeable only at $48 \mathrm{~h}$ (Supplementary Fig. 2c,d). Furthermore, the inhibitory effect of Ubp was minor compared to that of hCAML in spite of higher levels of Ubp expression in the cells (Supplementary Fig. 2d).

\section{Vpu and HIV-2 Env overcome the CAML block of virus release}

Like Vpu, HIV-2 Env enhances HIV-1 release by counteracting a cellular restriction unique to the human host $10,11,14$. To determine whether CAML is the restriction factor that confers the Vpu- and HIV-2 Env-dependent viral release phenotype in Cos-7 cells, we tested HIV-1 Gag release from Cos-7 cells expressing hCAML and Vpu or HIV-2 Env (Fig. 3a, b). Of note, the effect of Vpu on HIV-1 particle release is independent of the expression of the viral envelope glycoprotein ${ }^{20}$. HIV-1 Gag was expressed via a plasmid that encodes HIV-1 GagPol and Rev, and hCAML was expressed as EGFP-CAML, a fusion protein that maintains wild-type CAML restriction activity. Cells and supernatants were assayed for viral p24 content at 12, 24 and $48 \mathrm{~h}$ after transfection, and results were expressed as percentage Gag release (Fig. 
3a). Intracellular protein expression was evaluated by immunoblotting of the cell lysates (48 h) with the specific antibodies indicated in Figure 3b. Consistent with the previous findings, Cos-7 cells showed efficient release of HIV-1 Gag in the absence of Vpu (98\%), and Vpu or HIV-2 Env had no additional effect on Gag release (Fig. 3a). However, in Cos-7 cells expressing EGFP-CAML, there was a progressive reduction in Gag release to a minimum of $6 \%$ at $48 \mathrm{~h}$ (Fig. 3a). Total Gag protein in these cells was not diminished (data not shown).

Notably, expression of Vpu or Env in the presence of EGFP-CAML restores Gag release (92\% or $98 \%$, respectively). These results indicate that Vpu and HIV-2 Env, when expressed individually, are able to overcome the hCAML-mediated inhibition of Gag release, suggesting that these two viral proteins act to overcome a common CAML-mediated restriction to viral release.

\section{Rescue of $\mathrm{vpu}^{-}$HIV release in CAML-depleted human cells}

We next tested the effect of RNAi-mediated depletion of endogenous CAML on HIV-1 Gag or NLUdel viral release from human cells. Both HeLa and E6-1 T cells require Vpu for efficient viral release $10,12,21$. We first examined Gag particle release from HeLa cells transfected with varying concentrations of CAML-specific short interfering RNA (siRNA) duplexes or with control nontargeting siRNA (Fig. 4a). We assayed CAML depletion by immunoblotting of the cell lysates with CAML-specific antibodies (Fig. 4b). We noted that as intracellular CAML expression declined with rising amounts of siRNA, there was a concomitant dose-dependent increase in Gag release (Fig. 4a, b). We extended these studies to E6-1 cells by using a plasmid expressing GFP and short hairpin RNA (shRNA) that specifically targets CAML (shCAML). Release of NL4-3 or NLUdel virus was tested in HeLa cells (Fig. 4c, d) and E6-1 cells (Fig. $5 \mathrm{a}, \mathrm{b}$ ). As expected, almost $80 \%$ of wild-type NL4-3 virus produced in either cell type was found in the supernatant by $2 \mathrm{~d}$ after infection (Figs. $4 \mathrm{c}$ and $5 \mathrm{a}$ ). In contrast, the mutant NLUdel virus release was severely impaired, and Vpu expression rescued its release (Figs. 4c and 5a). We noticed a marked increase in NLUdel virus release in cells transfected with shCAML construct (88\%), but not in the control-transfected cells. The levels were comparable to that seen with Vpu coexpression (Figs. 4c and 5a). The accompanying blots show successful depletion of CAML to near complete levels in HeLa cells (less so in E6-1 cells) transfected with shCAML, but not in the control-transfected cells (Figs. $4 \mathrm{~d}$ and $5 \mathrm{~b}$ ).

To confirm the specificity of the hCAML block, we expressed a myc-tagged shRNA-resistant CAML (myc-hCAML-R) in CAML-depleted HeLa cells and assayed its effect on HIV Gag release (Supplementary Fig. 4 online). Expression of myc-hCAML-R in these cells led to considerable restoration of CAML activity revealed by diminished Gag in the supernatants (Supplementary Fig. 4a and 4b). This confirms that hCAML itself confers the Vpu-responsive block to viral release in human cells (Figs. 4 and 5).

It has previously been shown that deletion of Vpu results in inefficient release of virions from the cell surface with resultant accumulation of viral particles at various stages of budding 8 . Therefore, we analyzed release of NLUdel from CAML-depleted (by shRNA) or control A3.01 lymphocytic cells by electron microscopy (EM). The A3.01 cells transfected with the control vector revealed a striking 'Vpu defect' marked by aggregation of viral particles at the plasma membrane (Fig. 5c, d). These virions were sometimes identified in strings attached to the membrane (Fig. 5c). However, CAML depletion in A3.01 cells relieved the Vpu defect, as shown by efficient virion release and lack of accumulated viral particles at the cell surface (Fig. 5d). These results indicate that the phenotype of mature particle retention at the cell surface, which is characteristic of the Vpu-sensitive block to HIV-1 release, is reversed by depletion of CAML. 


\section{Human CAML restricts MLV release}

Retroviruses such as MLV and EIAV differ from HIV-1 in their interactions with components of the endosomal sorting complex required for transport (ESCRT) required for late budding events $22-25$. However, they encounter a common cellular restriction in human cells, demonstrated by their need for HIV-1 Vpu or HIV-2 Env for efficient release ${ }^{11,12}$. To test whether hCAML exerts a common block to retroviral egress from human host cells, we assayed MLV Gag release from both restrictive HeLa and permissive Cos-7 cells (Fig. 6a, b). MLV Gag was expressed with or without Vpu in cells pretransfected with control vector or vector expressing CAML shRNA. Two days after transfection, cell and purified viral lysates were analyzed for MLV Gag p65 and p30 expression by immunoblotting with CA-specific antibodies. Membranes were reprobed for endogenous CAML and for $\beta$-actin as a loading control (Fig. 6a). Cells were restricted in MLV Gag release in the absence of Vpu, with low levels of viral capsid in the supernatants (Fig. 6a). Vpu expression resulted in a marked increase in MLV Gag release (Fig. 6a). Depletion of endogenous CAML from HeLa cells also led to enhanced virus release (Fig. 6a). The cell-associated p65 Gag abundance (Fig. 6a) revealed that the enhanced particle release was indeed due to the reduction in intracellular CAML expression but not due to the increased Gag expression in the cells. The control vector alone did not have any effect on the intracellular CAML abundance or on MLV Gag release (Fig. $6 a)$.

We also evaluated MLV Gag release from Cos-7 cells expressing hCAML (Fig. 6b). As with HIV, a considerable amount of MLV Gag was released from Cos-7 cells in the absence of Vpu (p30), and Vpu expression did not enhance MLV output (Fig. 6b). However, hCAML expression interfered with the MLV Gag release from Cos cells, as shown by the reduced amount of capsid in the supernatant (Fig. 6b). Of note, MLV output in hCAML-expressing Cos-7 cells was rescued via expression of $\mathrm{Vpu}$, as shown by an amplified p30 signal in the cellular supernatants (Fig. 6b). Vpu expression was confirmed in both cell types by immunoblotting (data not shown). Taken together, these experiments suggest that the CAMLmediated block to viral release is not restricted to HIV-1, but also extends to MLV, consistent with one of the known features of the Vpu-responsive human restriction to particle release.

\section{DISCUSSION}

Here we identify CAML as a host cell restriction factor that is inhibited by Vpu. CAML expression in cells that are normally permissive for particle release and unresponsive to Vpu rendered them restrictive and $\mathrm{Vpu}$ responsive. Depletion of CAML in restrictive cells conferred a permissive and Vpu-independent phenotype. Furthermore, CAML-mediated restriction could be overcome by the action of HIV-2 ${ }_{\text {ROD } 10}$ Env, which counteracts restriction to particle release in a Vpu-like manner. Thus CAML fits very well the characteristics of the dominant, Vpuresponsive human host restriction factor predicted by earlier studies performed in humansimian heterokaryons ${ }^{10}$. These findings suggest that a major function of the HIV-1 Vpu protein is to counteract the CAML-mediated block to particle release.

The mechanism by which Vpu regulates viral release is distinct from that of the viral late domain-ESCRT interaction 9,18 . In the absence of $\mathrm{Vpu}$, mature virus particles are able to bud but remain tethered to the surface of infected cells, where they can be removed from the cell surface with protease treatment ${ }^{18}$. Consistent with this, virus particles retained in CAMLexpressing Cos-7 cells were recovered by treatment with protease (Supplementary Fig. 5 online). The mechanism by which CAML elicits this phenotype is not yet known. Vpu is present within the pericentriolar recycling endosome and must exit this compartment to exert its effects on particle release ${ }^{14}$. In this light, it is noteworthy that CAML is required for efficient recycling of epidermal growth factor receptor to the plasma membrane ${ }^{17}$. We detected colocalization between CAML and Vpu within perinuclear compartments, suggesting that CAML and Vpu 
may interact along the recycling pathway. Interaction with Vpu may prevent CAML from reaching the plasma membrane itself or, alternatively, may prevent CAML from directing the recycling of another factor involved in particle retention. We note that during the review of this manuscript, a protein known as BST-2 (or HM1.24) was shown to restrict HIV particle release and was termed 'tetherin' for this effect ${ }^{26}$. Tetherin meets many of the criteria for a cellular restriction factor that inhibits virus release, including differential expression in human cells that correlates well with the restrictive, Vpu-responsive cellular phenotype. We do not yet know if CAML alters the cell surface expression of tetherin or other aspects of its activity, but we do believe that the potential relationship between CAML and tetherin is worthy of investigation. Notably, both tetherin and CAML are targets for gene products of Kaposi's sarcoma-associated herpes virus (KSHV). Cellular levels of tetherin are decreased by the K5 gene product of $\mathrm{KSHV}^{27}$, and the $\mathrm{K} 7$ gene product of KSHV directly interacts with CAML to regulate cytosolic $\mathrm{Ca}^{2+}$ levels and thereby to maintain persistent viral infection in the host $^{28}$.

In contrast to hCAML, African green monkey CAML (agmCAML) was unable to block $v p u^{-}$particle release. Lack of expression of agmCAML does not seem to account for the difference, as CAML was readily detected in Cos-7 cells (Supplementary Fig. 3b). However, we noticed a weaker interaction of AGM CAML with Vpu in co-IP studies, whereas hCAML interacted more efficiently (Supplementary Fig. 6 online). Preliminary work has also identified genetic polymorphisms that distinguish agmCAML from hCAML (data not shown). Future work is required to completely define this species-specific difference in restriction, as well as to delineate the precise mechanism by which Vpu overcomes CAML-mediated restriction of particle release.

\section{METHODS}

\section{Cell lines and transfections}

HeLa, HOS and Cos-7 cells were obtained from American Type Culture Collection. Jurkat E6-1 and A3.01 cells were obtained from A. Weiss and T. Folks, respectively, through the US National Institutes of Health (NIH) AIDS Research and Reference Reagent Program. Cells were maintained under the recommended culture conditions. We transfected cells with Lipofectamine (Invitrogen) or electroporation (Cytopulse) following the manufacturer's protocols. When required, we isolated viable E6-1 or A3.01 cells by gradient centrifugation on FICOLL-HIPAQUE (Amersham).

\section{Plasmids}

We engineered plasmids pCMV-HACAML, pCMV-HAUbp and pCDNA-VpuHA using standard molecular biology protocols (available upon request). EGFP-CAML has been described elsewhere ${ }^{29}$. We generated The pCMS-H1 hCAML plasmid expressing shCAML by cloning the annealed oligonucleotides 1 (5'-GATCCCCGAGCTGAGCTAGTGCTTTTTT CAAGAGAAAAAGCACTAGCTCAGCTCTTTTTGGAAA-3') and 2 (5'-AG CTTTTCCAAAAAGAGCTGAGCTAGTGCTTTTTCTCTTGAAAAAAGCACT AGCTCAGCTCGGG-3') into the parent plasmid pCMS3.eGFP.H1p, provided by R. Bram and D. Billadeau ${ }^{30}$. The siRNA-resistant myc-human CAML (myc-hCAML-R) expression plasmid is similar to pCMV-HACAML; both lack the $3^{\prime}$ untranslated region targeted by the shRNA. We obtained plasmid pSV- $\psi-M L V-$ env $^{-}$from N. Landau through the US NIH AIDS Research and Reference Reagent Program. Plasmids pSVGAGPOL.rre and HIV-2 Env pRODA1 have been described elsewhere ${ }^{14,31}$. 


\section{Yeast two-hybrid screen}

We inserted full-length human codon-optimized Vpu cDNA in frame into pGBKT7 vector and transformed the vector into the yeast strain AH109 to screen a HeLa cDNA library according to the manufacturer's protocols (Clontech). We confirmed expression of the fusion protein by western blot analysis of total yeast extracts with monoclonal antibody to c-myc (Clontech) or rabbit antibodies to Vpu. Library plasmids were recovered and sequenced by standard methods.

\section{Viral release assays}

We infected Cos-7 or HeLa cells with VSV-G-pseudotyped NL4-3 or NLUdel viruses at a multiplicity of infection (MOI) of 1 by methods described elsewhere ${ }^{10}$. We assayed cells and media at various time points for viral Gag by p24-capture ELISA ${ }^{32}$. We calculated percentage viral release with the following formula: total (p24 content in supernatant/(total p24 in cells + supernatant) $\times 100$. We performed E6-1 experiments by infecting naive or sorted GFP-positive cells $\left(0.5 \times 10^{6} /\right.$ well $)$ with NL4-3 or NLUdel viruses at an MOI of 2 for $4 \mathrm{~h}$. Before sorting, we transduced cells with a retrovirus expressing Vpu. We filtered viral particles and pelleted them through a $20 \%$ sucrose cushion by centrifugation at $100,000 \mathrm{~g}$. We analyzed the resulting virus pellets and cell lysates by NuPAGE and immunoblotting with specific antibodies. Analysis included cells lysates normalized for total protein. We used the following antibodies in the assays mentioned above: antibodies to HA or c-myc (COVANCE), antibodies to HIV-2 2 ST gp120 (US NIH AIDS Reference and Reagent program), rabbit antibodies to GFP (Santa Cruz Biotechnology), monoclonal antibodies specific for $\beta$-actin (SIGMA), rabbit antibodies to CAML and goat polyclonal antibodies to MLV CA (obtained from R.J. Bram and S.P. Goff, respectively).

\section{Viral replication assays}

We transduced Cos-7 cells with a retrovirus expressing human CD4 with empty vector or pCMV-myc hCAML. The next day, we infected cells with NL4-3 or NLUdel viruses at an MOI of 0.01 and sampled the medium at $0,1,2,3,4$ and $5 \mathrm{~d}$ after infection for p24 antigen quantification. Results represent virus production from triplicate treatments performed in parallel.

\section{RNA interference}

We cultured HeLa cells transfected with plasmids expressing HIV-1 Gag and siGENOME SMART pool Human CAMLG or siGENOME non-targeting control siRNA (Dharmacon) for $48 \mathrm{~h}$ before collecting supernatants and cells for analysis. Alternatively, we infected HeLa or E6-1 cells transfected with CAML knockdown plasmids expressing shCAML, pCMS3.eGFP.H-hCAML or control pCMS3.eGFP.H1p with NL4-3 or NLUdel and assayed viral release at 12, 24 and $48 \mathrm{~h}$ after infection. In the shRNA-resistant CAML experiments, we transfected CAML-depleted HeLa cells with pCMV-myc hCAML-R plasmid and assayed virus release as mentioned above.

\section{Co-immunoprecipitation}

We lysed cells transfected with the indicated plasmids (Fig. 1b and Supplementary Fig. 6) or infected with HIV with lysis buffer ( $150 \mathrm{mM} \mathrm{NaCl}, 20 \mathrm{mM}$ Tris HCl, $0.1 \% \mathrm{NP}-40, \mathrm{pH}$ 7.4) and removed the nuclei by a 10-min low-speed centrifugation step. We incubated precleared cell lysates with or without $5 \mu \mathrm{l}$ of purified rabbit or mouse antibodies to CAML or irrelevant rabbit or mouse $\mathrm{IgG}$ for $1 \mathrm{~h}$ and for an additional hour with A/G-Sepharose beads (Santa Cruz Biotechnology) by gentle rotation at $4 \mathrm{C}$. We washed beads three times in lysis buffer with protease inhibitors, two times in a wash buffer ( $100 \mathrm{mM}$ Tris $\mathrm{pH}$ 7.4) with $500 \mathrm{mM} \mathrm{LiCl}$ before suspending in $1 \times$ LDS load buffer. We analyzed proteins by immunoblotting with either HA- 
or Vpu-specific antibodies. Before co-IP, we analyzed a $20-\mu 1$ aliquot of the lysates for $\mathrm{Vpu}$ and actin.

\section{Microscopy}

We stained cells on collagen-coated cover slips for endogenous CAML using protocols described elsewhere ${ }^{14}$. We analyzed images obtained with a laser-spinning confocal microscope (Perkin Elmer) with Volocity software package (Perkin Elmer-Improvision). For EM studies, we infected sorted GFP-positive A3.01 cells expressing shRNA expression or control plasmids with the indicated viruses (Fig. 5c, d) at an MOI of 1.5. After $62 \mathrm{~h}$ of culture, we fixed infected cells ( $2.5 \%$ glutaraldehyde in $100 \mathrm{mM}$ cacodylate buffer), embedded them in Eponate 12 and sectioned and stained them for transmission EM analysis on a Hitachi H-7500 transmission electron microscope.

\section{Statistical analyses}

Statistical analysis of colocalized pixels to derive Pearson's coefficient of colocalization was performed with Volocity 4.2 software (Improvision), as outlined in the legend to Supplementary Figure 1.

\section{Supplementary Material}

Refer to Web version on PubMed Central for supplementary material.

\section{Acknowledgements}

CA-specific antibodies were a gift from S. Goff (Columbia University). A. Weiss and T. Folks (US NIH AIDS Research and Reference Program) provided HeLa, Cos-7, Jurkat E6-1 and A3.01 cells. R. Bram and D. Billadeau (Mayo Clinic) provided plasmid pCMS3.eGFP.H1p. N. Landau (New York University) provided plasmid plasmid pSV- $\psi$-MLVenv $^{-}$. This research was supported by grants from Vanderbilt-Meharry Center for AIDS Research Developmental Core funded by the US NIH (to V.V.), the Vanderbilt Department of Pediatrics (to V.V.), Building Interdisciplinary Research Careers in Women's Health (to V.V.) and the US NIH (AI058828 to P.S. and 5R01CA112414 to R.J.B). We thank S.P. Goff (Columbia University) and K. Strebel (NIH) for reagents used in the study. We thank the Center for AIDS Research cell immunopathogenesis core and Emory School of Medicine EM core for help. We also thank R.T. D'Aquila and H.E. Ruley for critical review of the manuscript.

\section{References}

1. Strebel K, Klimkait T, Martin MA. A novel gene of HIV-1, vpu, and its 16-kilodalton product. Science 1988;241:1221-1223. [PubMed: 3261888]

2. Maldarelli F, Chen MY, Willey RL, Strebel K. Human immunodeficiency virus type 1 Vpu protein is an oligomeric type I integral membrane protein. J Virol 1993;67:5056-5061. [PubMed: 8331740]

3. Willey RL, Maldarelli F, Martin MA, Strebel K. Human immunodeficiency virus type 1 Vpu protein induces rapid degradation of CD4. J Virol 1992;66:7193-7200. [PubMed: 1433512]

4. Terwilliger EF, Cohen EA, Lu YC, Sodroski JG, Haseltine WA. Functional role of human immunodeficiency virus type 1 vpu. Proc Natl Acad Sci USA 1989;86:5163-5167. [PubMed: 2472639]

5. Margottin F, et al. A novel human WD protein, h- $\beta$ TrCp, that interacts with HIV-1 Vpu connects CD4 to the ER degradation pathway through an F-box motif. Mol Cell 1998;1:565-574. [PubMed: 9660940]

6. Bai C, et al. SKP1 connects cell cycle regulators to the ubiquitin proteolysis machinery through a novel motif, the F-box. Cell 1996;86:263-274. [PubMed: 8706131]

7. Schubert U, Clouse KA, Strebel K. Augmentation of virus secretion by the human immunodeficiency virus type $1 \mathrm{Vpu}$ protein is cell type independent and occurs in cultured human primary macrophages and lymphocytes. J Virol 1995;69:7699-7711. [PubMed: 7494279] 
8. Klimkait T, Strebel K, Hoggan MD, Martin MA, Orenstein JM. The human immunodeficiency virus type 1-specific protein vpu is required for efficient virus maturation and release. J Virol 1990;64:621629. [PubMed: 2404139]

9. Schwartz MD, Geraghty RJ, Panganiban AT. HIV-1 particle release mediated by Vpu is distinct from that mediated by p6. Virology 1996;224:302-309. [PubMed: 8862425]

10. Varthakavi V, Smith RM, Bour SP, Strebel K, Spearman P. Viral protein U counteracts a human host cell restriction that inhibits HIV-1 particle production. Proc Natl Acad Sci USA 2003;100:1515415159. [PubMed: 14657387]

11. Abada P, Noble B, Cannon PM. Functional domains within the human immuno-deficiency virus type 2 envelope protein required to enhance virus production. J Virol 2005;79:3627-3638. [PubMed: 15731257]

12. Gottlinger HG, Dorfman T, Cohen EA, Haseltine WA. Vpu protein of human immunodeficiency virus type 1 enhances the release of capsids produced by gag gene constructs of widely divergent retroviruses. Proc Natl Acad Sci USA 1993;90:7381-7385. [PubMed: 8346259]

13. Bour S, Strebel K. The human immunodeficiency virus (HIV) type 2 envelope protein is a functional complement to HIV type $1 \mathrm{Vpu}$ that enhances particle release of heterologous retroviruses. J Virol 1996;70:8285-8300. [PubMed: 8970948]

14. Varthakavi $\mathrm{V}$, et al. The pericentriolar recycling endosome plays a key role in $\mathrm{Vpu}$-mediated enhancement of HIV-1 particle release. Traffic 2006;7:298-307. [PubMed: 16497224]

15. Bram RJ, Crabtree GR. Calcium signalling in T cells stimulated by a cyclophilin B-binding protein. Nature 1994;371:355-358. [PubMed: 7522304]

16. Tran DD, et al. CAML is a p56Lck-interacting protein that is required for thymocyte development. Immunity 2005;23:139-152. [PubMed: 16111633]

17. Tran DD, Russell HR, Sutor SL, van Deursen J, Bram RJ. CAML is required for efficient EGF receptor recycling. Dev Cell 2003;5:245-256. [PubMed: 12919676]

18. Neil SJ, Eastman SW, Jouvenet N, Bieniasz PD. HIV-1 Vpu promotes release and prevents endocytosis of nascent retrovirus particles from the plasma membrane. PLoS Pathog 2006;2:e39. [PubMed: 16699598]

19. Callahan MA, et al. Functional interaction of human immunodeficiency virus type $1 \mathrm{Vpu}$ and Gag with a novel member of the tetratricopeptide repeat protein family. J Virol 1998;72:5189-5197. [PubMed: 9573291]

20. Yao XJ, Gottlinger H, Haseltine WA, Cohen EA. Envelope glycoprotein and CD4 independence of vpu-facilitated human immunodeficiency virus type 1 capsid export. J Virol 1992;66:5119-5126. [PubMed: 1629967]

21. Cervantes-Acosta G, Cohen EA, Lemay G. Human Jurkat lymphocytes clones differ in their capacity to support productive human immunodeficiency virus type 1 multiplication. J Virol Methods 2001;92:207-213. [PubMed: 11226568]

22. Garrus JE, et al. Tsg101 and the vacuolar protein sorting pathway are essential for HIV-1 budding. Cell 2001;107:55-65. [PubMed: 11595185]

23. Martin-Serrano J, Zang T, Bieniasz PD. Role of ESCRT-I in retroviral budding. J Virol 2003;77:47944804. [PubMed: 12663786]

24. Tanzi GO, Piefer AJ, Bates P. Equine infectious anemia virus utilizes host vesicular protein sorting machinery during particle release. J Virol 2003;77:8440-8447. [PubMed: 12857913]

25. Strack B, Calistri A, Craig S, Popova E, Gottlinger HG. AIP1/ALIX is a binding partner for HIV-1 p6 and EIAV p9 functioning in virus budding. Cell 2003;114:689-699. [PubMed: 14505569]

26. Neil SJ, Zang T, Bieniasz PD. Tetherin inhibits retrovirus release and is antagonized by HIV-1 Vpu. Nature 2008;451:425-430. [PubMed: 18200009]

27. Bartee E, McCormack A, Fruh K. Quantitative membrane proteomics reveals new cellular targets of viral immune modulators. PLoS Pathog 2006;2:e107. [PubMed: 17238276]

28. Feng P, et al. Kaposi's sarcoma-associated herpesvirus mitochondrial K7 protein targets a cellular calcium-modulating cyclophilin ligand to modulate intra-cellular calcium concentration and inhibit apoptosis. J Virol 2002;76:11491-11504. [PubMed: 12388711] 
29. Tovey SC, Bootman MD, Lipp P, Berridge MJ, Bram RJ. Calcium-modulating cyclophilin ligand desensitizes hormone-evoked calcium release. Biochem Biophys Res Commun 2000;276:97-100. [PubMed: 11006089]

30. Gomez TS, et al. HS1 functions as an essential actin-regulatory adaptor protein at the immune synapse. Immunity 2006;24:741-752. [PubMed: 16782030]

31. Smith AJ, Cho MI, Hammarskjold ML, Rekosh D. Human immunodeficiency virus type 1 Pr55gag and Pr160gag-pol expressed from a simian virus 40 late replacement vector are efficiently processed and assembled into viruslike particles. J Virol 1990;64:2743-2750. [PubMed: 1692347]

32. Wehrly K, Chesebro B. p24 antigen capture assay for quantification of human immunodeficiency virus using readily available inexpensive reagents. Methods 1997;12:288-293. [PubMed: 9245608] 
a

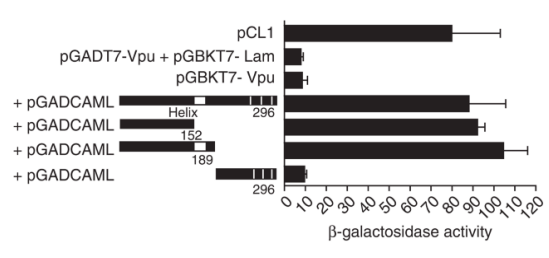

b

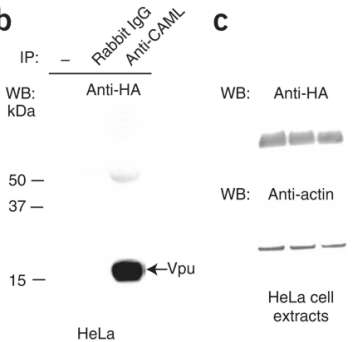

d

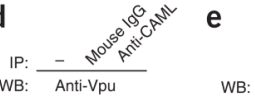

WB: Anti-Vpu

WB:

$50-$

$37-\quad--E \quad$ WB:

WB: Anti-actin

$15-$

$10-$

E6-1 E6-1 cell
extracts

Figure 1.

Interaction of HIV-1 Vpu with human CAML. (a) AH109 yeast transformed with the indicated control plasmids (top two plasmids) or pGBKT7-Vpu (bait) and pGADT7-CAML or pGADT7 encoding CAML truncation mutants (prey) were analyzed for protein-protein interactions in a liquid culture $\beta$-gal assay using chlorophenol red $\beta$-D-galactopyranoside as substrate. Results represent mean \pm s.d. from three independent experiments. (b-e) Co-immuno-precipitation analysis of Vpu-CAML interactions. Precleared cell extracts from HeLa cells transfected with pcDNA-VpuHA (b) or E6-1 cells infected with wild type NL4-3 (d) were incubated with prewashed protein $\mathrm{A} / \mathrm{G}+$ beads, beads conjugated to irrelevant mouse or rabbit $\mathrm{IgG}$ or beads conjugated to CAML-specific antibodies (anti-CAML). The resulting complexes were analyzed by immunoblotting with antibodies to HA (anti-HA, b) or Vpu (anti-Vpu, d). 20- $\mu$ l aliquots of the representative cell lysates were also analyzed in parallel for VpuHA (c) or Vpu (e) and actin. WB, western blot. IP, immunoprecipitation. 
a

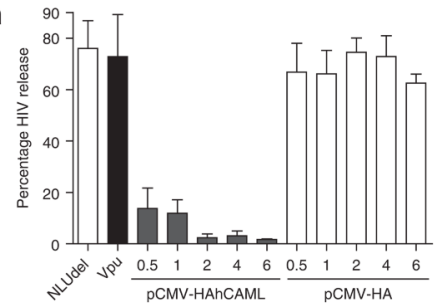

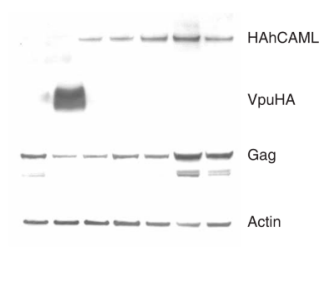

C
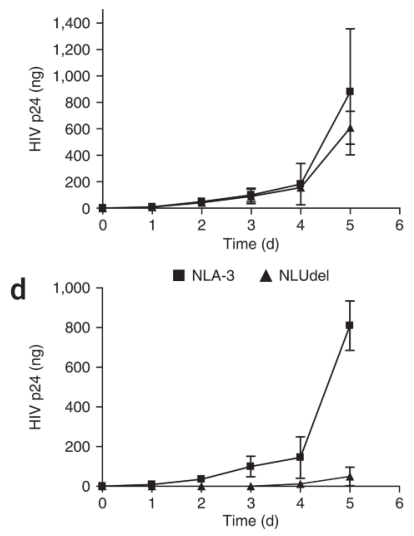

Figure 2.

Human CAML blocks Vpu deletion mutant viral release in Cos-7 cells. (a) Cos-7 cells transfected with the indicated amounts (in $\mu \mathrm{g}$ ) of control pCMV-HA or pCMV-HACAML plasmids were infected with NLUdel virus. After $48 \mathrm{~h}$, cells and supernatants were analyzed for Gag in a p24 ELISA. Results represent mean \pm s.d. from triplicate transfections. (b) Cos cell lysates from a were probed for CAML or Vpu by immunoblotting with HA-specific antibodies and for HIV Gag with CA-specific antibodies. Blots were reprobed for $\beta$-actin with actin-specific monoclonal antibodies. (c-d) hCAML blocks $v p u^{-}$mutant virus production from Cos-7 cells expressing human CD4. Cos-7 cells transfected with a construct, pCMV-myc (c) or pCMV-mycCAML (d), were transduced with a VSV-G-pseudotyped retrovirus expressing CD4. After an overnight culture, cells were infected with wild-type NL4-3 or NLUdel viruses at an MOI of 0.01. Four hours later, cells were washed and cultured in growth medium for 0 , 1, 2, 3, 4 and $5 \mathrm{~d}$. Samples were assayed daily for HIV release by p24 ELISA. Results represent mean \pm s.d. from triplicate independent experiments performed in parallel. 
a

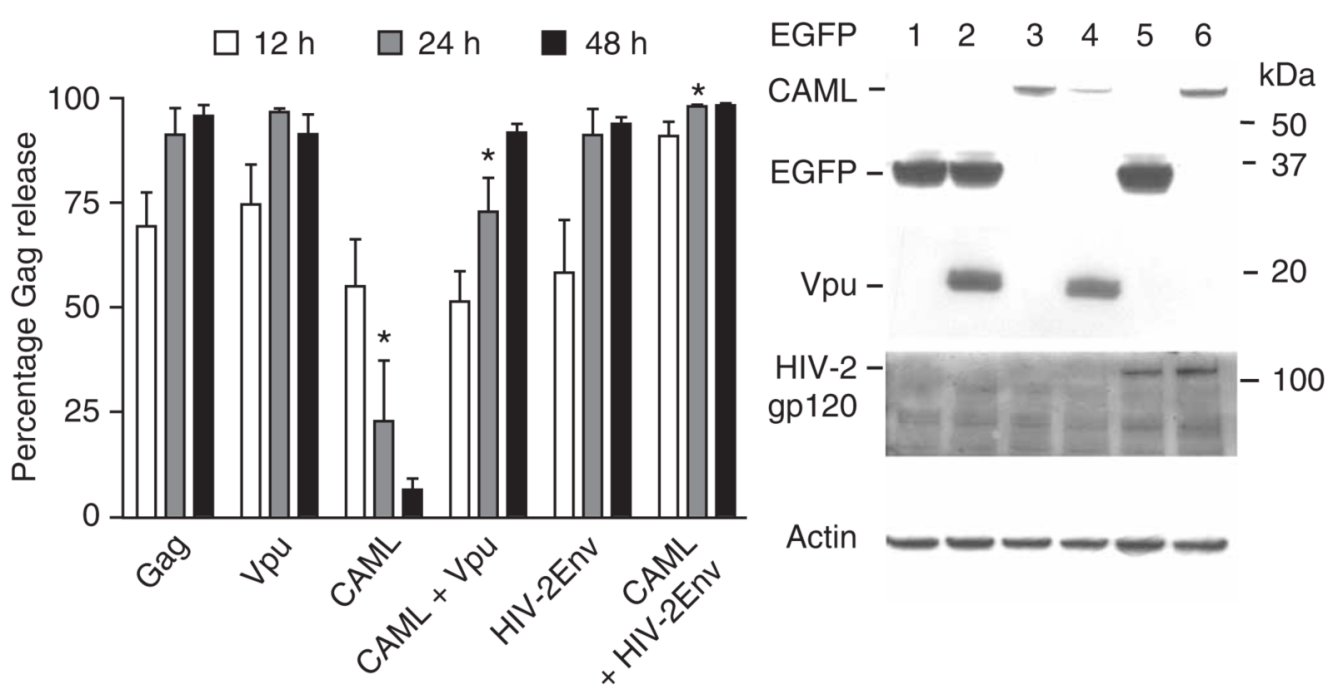

Figure 3.

Vpu and HIV-2 Env overcome an hCAML-induced block to viral release in Cos-7 cells. (a) Cos-7 cells were transfected with a Gag expression plasmid pSVGAGPOL.rre (Gag) alone or cotransfected with pcDNA-VpuHA (Vpu) or pROD-A1 (HIV-2 Env). CAML was expressed via $\mathrm{pEGFP-CAML}$, and transfections were normalized by the addition of vector DNA (pEGFPC1). The transfections that included EGFP-CAML are marked with asterisks. Cells expressing the indicated proteins were analyzed for Gag release at 12, 24 and $48 \mathrm{~h}$ of culture. (b)

Immunoblotting analysis of CAML and viral proteins from the 48-h cell lysates from a. The numbers 1-6 correspond to the treatments in order from left to right in the bar graph in $\mathbf{a}$. EGFP and EGFP-CAML were detected with GFP-specific antibodies. Membranes were reprobed for VpuHA, HIV-2 Env and $\beta$-actin with antibodies to HA, HIV-2 gp120 (the HIV-2 Env surface (SU) component) or actin. 

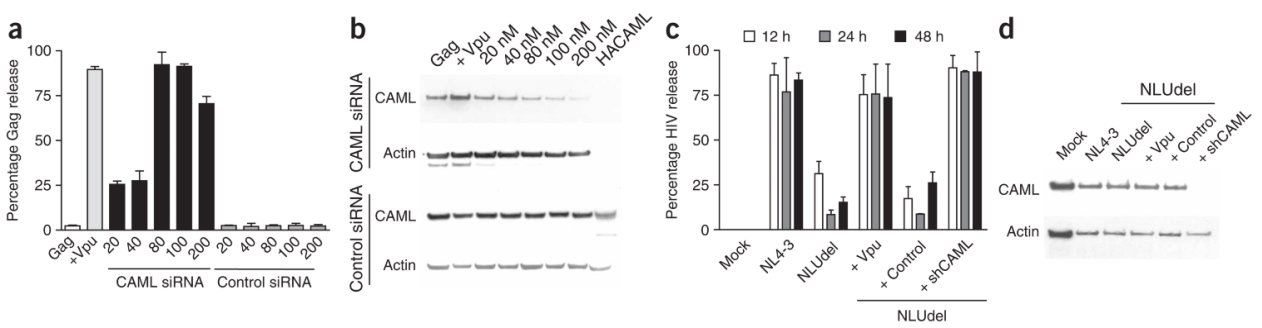

Figure 4.

CAML depletion eliminates the Vpu-sensitive cellular block to viral release in restrictive $\mathrm{HeLa}$ cells. (a) HeLa cells transfected with a Gag expression plasmid, pSVGAGPOL.rre, and the indicated amounts (in $\mathrm{nM}$ ) of a pool of CAML-specific or non-targeting siRNA were analyzed for Gag release at $48 \mathrm{~h}$ by p24 ELISA. Results represent mean \pm s.d. derived from triplicate transfections. (b) Cell lysates normalized for total protein were analyzed for CAML depletion via immunoblotting with rabbit antibodies to CAML. (c) HeLa cells transfected with pCMS3eGFP.H1p (control), pCMS3.eGFP.H-hCAML (shCAML) or VpuHA were infected with wildtype NL4-3 or NLUdel viruses at an MOI of 1. Cells and supernatants at 12, 24 and $48 \mathrm{~h}$ after infection were analyzed for viral capsid. (d) Cell lysates from $\mathbf{c}$ were normalized for total protein and tested for CAML depletion by immunoblotting with CAML- and actin-specific antibodies. 
a

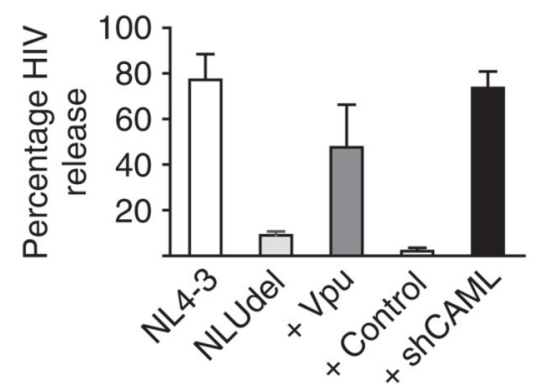

b

C

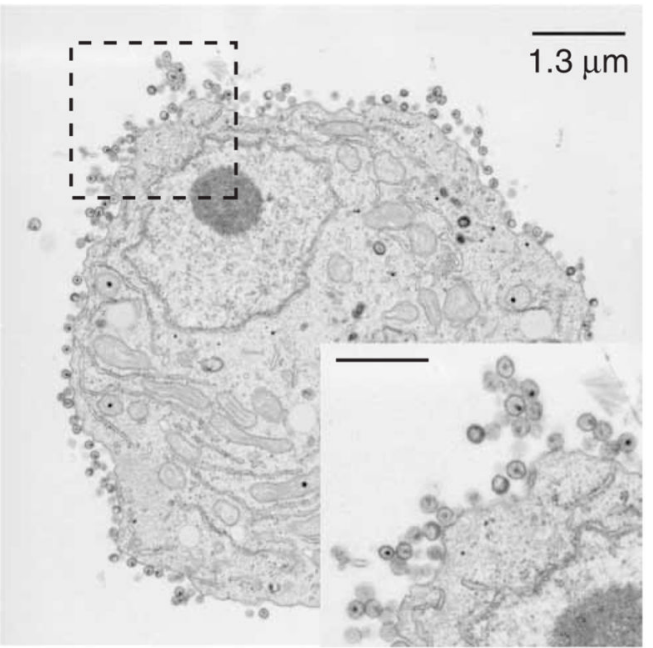

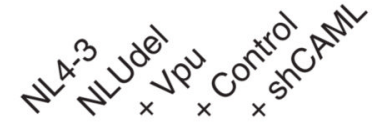

CAML

\section{Actin}

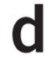

A3.01-shCAML

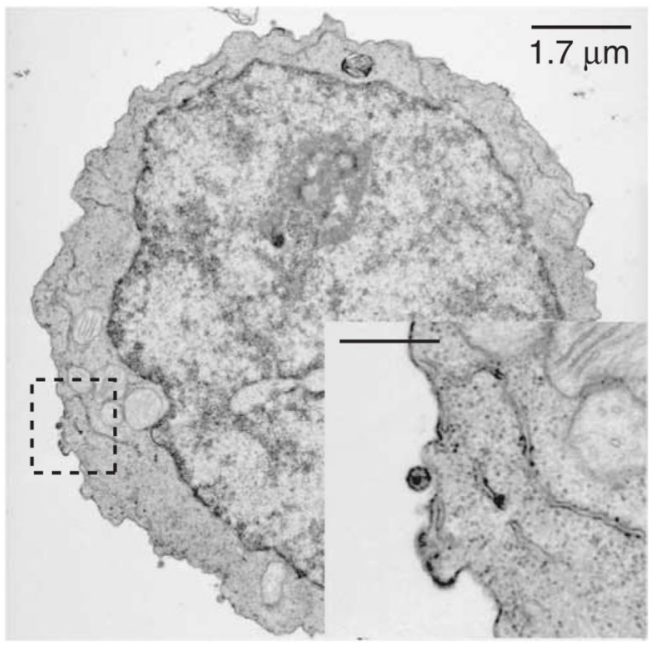

Figure 5.

Depletion of endogenous CAML from the E6-1 T cell line rescues $v p u^{-}$mutant viral release. (a) E6-1 lymphocytes were transfected with the control or shCAML expression plasmids by electroporation. One day later, GFP-positive cells were sorted from both of the transfections and then infected with NLUdel virus at an MOI of 2, and viral release was assayed at $48 \mathrm{~h}$ after infection. For Vpu expression, cells were transduced with a recombinant retrovirus expressing $\mathrm{Vpu} 4 \mathrm{~h}$ before sorting. (b) Cell extracts were normalized for total protein before immunoblotting for CAML. (c,d) Analysis of NLUdel viral release phenotype in CAMLdepleted lymphocytic cells by EM. A3.01 cells were transfected with the control (c) or shCAML (d) plasmids by electroporation. GFP-positive cells were sorted and infected with NLUdel virus, and the viral release phenotype was analyzed by EM. Quantification of surfaceassociated particles revealed that 35 of 81 (43\%) NLUdel-infected cells receiving the control plasmid had > 25 particles on the plasma membrane, compared with only 1 of 61 infected cells receiving the CAML shRNA. For this analysis, 25 particles represented two standard deviations above the mean value for NL4-3-infected control cells. Scale bars in the insets, $0.7 \mu \mathrm{m}(\mathbf{c})$ and $0.5 \mu \mathrm{m}(\mathbf{d})$. 
a

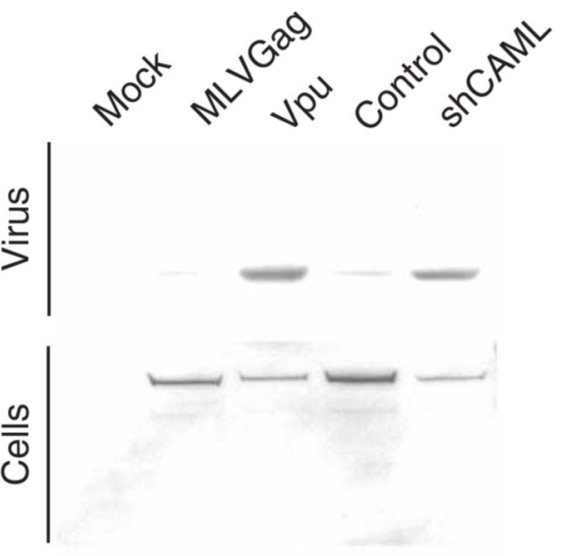

CAML

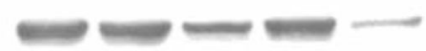

Actin b

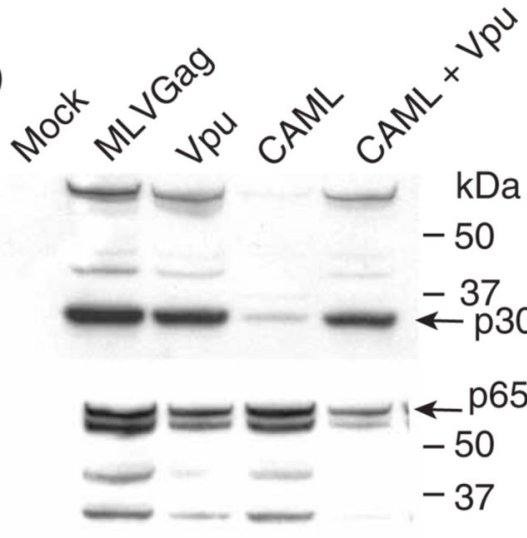

EGFP, CAML $-50$

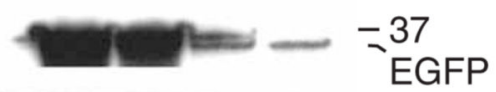

\section{Figure 6.}

CAML restricts MLV release. (a) HeLa cells transfected with control vector, shCAML vector or pcDNA-VpuHA (Vpu) as indicated were cultured overnight to allow protein expression. The next day, cells were transfected with $10 \mu \mathrm{g}$ of MLV Gag expression plasmid, pSV- $\psi$ MLV-env ${ }^{-}$. Two days later, concentrated viral and cell lysates were analyzed for MLV Gag p65 in cells and p30 in supernatants by immunoblotting with goat antisera to MLV CA. CAML depletion in cells was assayed with CAML-specific antibodies. (b) Cos-7 cells expressing EGFP, EGFP-CAML or Vpu were transfected with the pSV- $\psi$-MLV-env ${ }^{-}$. MLV Gag in the supernatants and cells was analyzed as in a. EGFP and EGFP-CAML were detected with GFPspecific antibodies. As a control, mock-transfected HeLa or Cos-7 cells were assayed in parallel with the rest of the treatments in $\mathbf{a}$ and $\mathbf{b}$. 